When Wanting the Best Goes Right or Wrong: Distinguishing Between Adaptive and Maladaptive Maximization

Jeffrey Hughes \& Abigail A. Scholer

University of Waterloo

Correspondence should be addressed to Jeffrey Hughes, Department of Psychology, University of Waterloo, 200 University Ave. W, Waterloo, Ontario, Canada, N2L 3G1, j4hughes@uwaterloo.ca; or Abigail Scholer, Department of Psychology, Department of Psychology, University of Waterloo, 200 University Ave. W, Waterloo, Ontario, Canada, N2L 3G1, ascholer@uwaterloo.ca. 


\begin{abstract}
Researchers have often disagreed on how to define maximization, leading to conflicting conclusions about its potential benefits or drawbacks. Drawing from motivation research, we distinguish between the goals (i.e., wanting the best) and strategies (e.g., alternative search) associated with maximizing. Three studies illustrate how this differentiation offers insight into when maximizers do or do not experience affective costs when making decisions. In Study 1, we show that two motivational orientations, promotion focus and assessment mode, are both associated with the goal of wanting the best, yet assessment (not promotion) is related to the use of alternative search strategies. In Study 2, we demonstrate that alternative search strategies are associated with frustration on a discrete decision task. In Study 3, we provide evidence that one reason for this link may be due to reconsideration of previously dismissed options. We discuss the potential of this approach to integrate research in this area.
\end{abstract}

Keywords: maximizing, decision-making, regulatory focus, regulatory mode, motivation 


\section{When Wanting the Best Goes Right or Wrong: Distinguishing Between Adaptive and Maladaptive Maximization}

People make innumerable decisions over the course of each day. Some of these decisions are trivial — what clothes to wear, what route to take to work - but others can be very significant to one's life-what career to pursue, what house to buy, whom to marry. The outcomes of these decisions, large or small, can have important consequences for our well-being. However, the processes we use to make these decisions can also impact our health, happiness, and satisfaction (e.g., Arkes \& Blumer, 1985; Hsee \& Hastie, 2006; Ratner, Kahn, \& Kahneman, 2014). Indeed, the decision-making experience, not just the decision outcomes, may influence well-being.

One approach to decision-making is to maximize, which is commonly defined as an extensive search through options to find the best one (Schwartz et al., 2002; Simon, 1955, 1956). At first glance, such an approach may appear to yield the best decisions (and some evidence suggests that it can; Iyengar, Wells, \& Schwartz, 2006); however, research has been mixed about the affective costs associated with the process or experience of maximizing. Some research has found associations between chronic maximizing and greater depression, lower self-esteem, and lower satisfaction with one's choices (Dar-Nimrod, Rawn, Lehman, \& Schwartz, 2009; Iyengar et al., 2006; Schwartz et al., 2002). However, other research has found no association between maximizing and these negative outcomes (Diab, Gillespie, \& Highhouse, 2008; Nenkov, Morrin, Ward, Schwartz, \& Hulland, 2008), or has even observed associations with positive outcomes, such as higher optimism and self-efficacy (Lai, 2010); and higher life satisfaction, subjective happiness, and positive affect (Purvis, Howell, \& Iyer, 2011). Thus, it is important to understand what underlies these conflicting findings- how the experience or process of maximizing may or may not be costly, irrespective of the quality of the decision made. We propose that the 
incongruities between these findings can be largely attributed to competing ideas about how to conceptualize maximizing as a construct.

\section{Past Work Measuring Maximization}

Research on maximization has generally focused on three factors: pursuit of the best option (generally referred to as "high standards"), extensively evaluating options ("alternative search"), and experiencing the process of making decisions as difficult ("decision difficulty;" Nenkov et al., 2008). Despite the fact that maximization was originally conceptualized as a unitary construct (Schwartz et al., 2002), subsequent research has suggested that the three factors described above are only moderately related (Nenkov et al., 2008; Purvis et al., 2011; Rim, Turner, Betz, \& Nygren, 2011). In addition, researchers have created a variety of scales to measure maximizing that are only loosely correlated with each other (Giacopelli, Simpson, Dalal, Randolph, \& Holland, 2013; Rim et al., 2011; Weinhardt, Morse, Chimeli, \& Fisher, 2012), in part because each emphasizes different facets of the construct (Diab et al., 2008; Lai, 2010; Turner, Rim, Betz, \& Nygren, 2012; Weinhardt et al., 2012). As a result, maximizing has been associated with both negative and positive outcomes (e.g., both lower and higher life satisfaction) depending on the particular scales and subscales used in a given study.

When these subscales are considered separately, however, more consistent patterns emerge. Across numerous studies the alternative search and decision difficulty subscales (positively correlated with one another; Giacopelli et al., 2013; Purvis et al., 2011; Rim et al., 2011; Turner et al., 2012; Weinhardt et al., 2012) have been associated almost universally with negative experiences. Both the alternative search and decision difficulty subscales have been associated with greater depression and lower optimism (Nenkov et al., 2008); lower subjective happiness (Nenkov et al., 2008; Purvis et al., 2011); lower life satisfaction and greater 
psychological distress (Purvis et al., 2011); greater procrastination, lower self-efficacy, and lower self-regard (Rim et al., 2011); and greater indecisiveness and avoidant decision-making (Weinhardt et al., 2012).

In contrast, the high standards subscale has shown very different patterns: either no association (Nenkov et al., 2008) or a negative association (Weinhardt et al., 2012) with depression; linked with greater life satisfaction and subjective happiness (Purvis et al., 2011; Weinhardt et al., 2012); no relation with psychological distress (Purvis et al., 2011) or procrastination (Rim et al., 2011); positively related to positive affect (Purvis et al., 2011), selfefficacy, and self-regard (Rim et al., 2011); and negatively correlated with indecisiveness and avoidant decision-making (Weinhardt et al., 2012).

These divergent results underline the need for greater conceptual clarity with regard to what maximization is and how to think about the relations among the maximization facets. In fact, the clear patterns between the subscales suggest that there may be considerable utility in differentiating separate types of maximizing in terms of the way people engage in and experience decision processes. That is, irrespective of decision outcome, there may be relatively more or less adaptive types of maximizing in terms of the affective costs associated with the decision process. Specifically, we propose that there may be a relatively more adaptive way of maximizing that is not associated with affective costs when decision-making and a relatively more maladaptive way of maximizing that makes individuals more prone to experiencing affective costs. We propose that by distinguishing between the goals and strategies of decisionmakers, we can identify different types of maximizers, leading to new insights about what it means to maximize. 


\section{Differentiating Goals and Strategies}

Drawing upon the language of motivation (goals and strategies; e.g., Higgins, 2011;

Kruglanski et al., 2002), we argue for a conceptual distinction to be made between what maximizers want and what maximizers do. Stated differently, it is possible to distinguish between the optimization goal of wanting the "best" (as opposed to settling for something that is "good enough") and the decision strategy of alternative search that maximizers may use. Taking this approach, we agree with others who have argued that the optimization goal is most central to the core definition of maximizing (Dalal, Diab, Zhu, \& Hwang, 2015; Diab et al., 2008;

Giacopelli et al., 2013; Weinhardt et al., 2012). For instance, Diab et al. (2008), in their paper offering an alternate scale of maximizing tendencies, argued that the concept of maximization was most closely associated with the "general tendency to pursue the identification of the optimal alternative" (Diab et al., 2008, p. 365), or in other words, the goal of finding the "best" option. However, while the optimization goal may be most central for defining maximizing, the strategies that maximizers use to pursue this goal may have important consequences for the affective costs they experience during the decision process. Thus, unlike researchers who have excluded alternative search from their conceptualization of maximizing (e.g., Dalal et al., 2015;

Diab et al., 2008), we argue that the measurement of alternative search strategies is still a critical component in understanding the experiences of maximizers.

Indeed, alternative search strategies may be one common way in which this optimization goal is pursued. However, unlike researchers who make alternative search a core component of maximizing (Lai, 2010; Nenkov et al., 2008; Schwartz et al., 2002; Turner et al., 2012) we argue that this is only one possible strategy for pursuing this goal. It may be possible to identify individuals who pursue a maximizing goal but do not engage in alternative search. Specifically, 
different underlying motivations for pursuing this goal may lead to differences in the strategies that are used to pursue it. Importantly, we argue that these differences in strategy use may be critical for distinguishing between adaptive versus maladaptive maximizing in terms of the affective costs associated with decision-making. Given the positive correlation between alternative search and chronic decision difficulty (in our conceptualization, a possible outcome of engaging in maximizing, not part of the construct itself), as well as between alternative search and other chronic negative outcomes, this might suggest that alternative search (as it has been measured) may be one of the more maladaptive strategies that maximizers use.

\section{Identifying What May Be Maladaptive about Alternative Search}

As mentioned above, research suggests that alternative search is a strategy associated with chronic negative outcomes. However, it is unclear at first glance what exactly would make alternative search so problematic. In particular, there is little research that has examined the affective costs of maximizing on discrete decisions, rather than assessing general, chronic tendencies. As such, it is important to explore alternative search in more detail to understand what aspects of this strategy, when played out from decision to decision, might be particularly likely to be linked to affective costs.

An examination of the items in the Maximization Scale (Schwartz et al., 2002) and the Maximization Inventory (Turner et al., 2012) suggests that alternative search, as measured in prior research, may capture a number of different facets. Several items suggest rather face-valid (and seemingly non-problematic) elements of exhaustive search: examination of many options (e.g., "I treat relationships like clothing: I expect to try a lot on before I get the perfect fit"); examination of options on multiple dimensions (e.g., "I will continue shopping for an item until it reaches all of my criteria"); or greater time spent evaluating options (e.g., "When shopping, I 
plan on spending a lot of time looking for something"). However, some items suggest a different flavor of exhaustive search: returning to re-evaluate options that one had previously eliminated

(e.g., "When I am in the car listening to the radio, I often check other stations to see if something better is playing, even if I'm relatively satisfied with what I'm listening to"). We propose that this element of alternative search—reconsidering previously dismissed options_ — could be particularly problematic. Conceptually, it could be related to rumination and indecisiveness, two concepts often linked to negative outcomes such as depression, obsessive-compulsive behavior, and low sense of mastery (Frost \& Shows, 1993; Nolen-Hoeksema, Larson, \& Grayson, 1999; Nolen-Hoeksema, 2000). Thus, although we make general predictions about the broad "alternative search" construct as it has been measured and described in previous research (Nenkov et al., 2008), we also explore the particular behaviors associated with this strategy that may be particularly maladaptive (i.e., affectively costly) in any given decision.

\section{Antecedent Factors of Maximizing}

By distinguishing between goals and strategies of decision-makers, we propose that there may be important motivational antecedents that predict relatively adaptive versus maladaptive forms of maximizing in terms of the affective costs associated with decision-making. In keeping with the notion that the optimization goal is integral to what it means to be a maximizer, we sought to identify motivational orientations that would lead to similar predictions for the adoption of a maximizing goal (optimization). However, in order to differentiate between types of maximizing, we identified motivational orientations that would differ in predictions for the use of alternative search as a strategy used to pursue a maximizing goal and thus, presumably, the affective costs associated with that strategy. The intent was not to be exhaustive, but illustrative. Using this process, we identified assessment mode (Higgins, Kruglanski, \& Pierro, 
2003) and promotion focus (Higgins, 1997) as two ideal motivational constructs. These motivational orientations have been used to predict and explain broad patterns of behavior from leadership styles (Benjamin \& Flynn, 2006; Stam, Van Knippenberg, \& Wisse, 2010) to consumer behaviors (Lin \& Huang, 2011; Pierro et al., 2013) to health-related decisions and outcomes (Leder, Florack, \& Keller, 2014; Rivis, Sheeran, \& Armitage, 2010). We will explain and make predictions about each construct in turn.

Assessment mode. Assessment mode is a motivational orientation concerned with evaluating and comparing entities, such as goals and means, in pursuit of doing the "right" thing (Higgins et al., 2003). It is also associated with prioritizing value over expectancy (Kruglanski et al., 2000). Thus, we predict that assessment will be positively associated with the optimization goal.

Assessment mode is also characterized by an emphasis on careful and comprehensive evaluation. Assessors prefer full comparison decision strategies (Avnet \& Higgins, 2003), generate many means for a given goal (Kruglanski et al., 2000), and take time to measure and evaluate alternatives on their relative quality (Mauro, Pierro, Mannetti, Higgins, \& Kruglanski, 2009). Consequently, we predicted that assessment would also be positively associated with high use of alternative search. In addition, prior work has shown that assessment is positively correlated with neuroticism, discomfort with ambiguity, and indecisiveness (Kruglanski et al., 2000), which may suggest that assessors should be more likely to experience high decision difficulty. Because of the adoption of this strategy and these likely outcomes, we therefore posited that assessors likely enact a less adaptive form of maximizing: They will be more likely to experience affective costs as they make decisions in their pursuit of the optimization goal. In 
this sense, assessment as a construct may predict the subtype of maximizing that the original Maximization Scale (Schwartz et al., 2002) intended to measure.

Promotion focus. Promotion focus is a motivational orientation concerned with advancement and growth goals (Higgins, 1997). Promotion-focused individuals strive to attain ideals and are particularly concerned with approaching gains and avoiding non-gains (Scholer \& Higgins, 2011; Zou \& Scholer, 2016). Because of this emphasis on advancement and gains, we predicted that, as with assessment, promotion focus would be positively associated with the optimization goal of wanting the best.

However, promotion focus is also associated with a preference for eager (vs. vigilant) strategies to achieve goals (Scholer \& Higgins, 2011). Eager strategies are approach-oriented strategies such as initiation of goals (Fuglestad, Rothman, \& Jeffery, 2008), searching for matches vs. mismatches (Crowe \& Higgins, 1997; Higgins et al., 2001), optimism (Grant \& Higgins, 2003), and prioritizing speed over accuracy (Förster, Higgins, \& Bianco, 2003). Thus, although promotion focus has been associated with global processing (Förster \& Higgins, 2005) and generating more alternatives (Liberman, Molden, Idson, \& Higgins, 2001), eager strategies such as an emphasis on speed and searching for matches should balance the time and effort spent on searching for alternatives in a decision-making scenario. Thus, we predicted that promotion focus should be unrelated to or even negatively associated with alternative search. As a consequence, we predicted that promotion-focused individuals may enact an adaptive form of maximizing. Given the correlation between alternative search and decision difficulty, promotionfocused individuals should be less likely to experience affective costs in their pursuit of the optimization goal. 


\section{The Present Research}

The current research examines how distinguishing between the goals and strategies of maximizers can shed light on whether they experience affective costs. One of the major contributions of this research is to move beyond the chronic, dispositional measures to examine affective costs at the situational level, using discrete decision tasks. First, we establish the association between the motivational orientations of promotion and assessment with the various components of chronic maximizing assessed in previous literature (Study 1). Then, we examine maximizing in a discrete decision-making task to demonstrate that these motivations predict distinct types of maximizing with regard to affective costs associated with the decision experience: frustration (Studies 2 and 3) and regret (Study 3). Finally, in Study 3 we examine the particular processes through which different components of alternative search manifest in a discrete decision scenario. We examine how a chronic measure of alternative search predicts discrete decision behaviors - evaluation of options and the reconsideration of options that were previously dismissed - and how in turn these behaviors differ when predicting frustration and post-choice regret.

\section{Study 1}

The major impetus of Study 1 was to establish how the motivational constructs of promotion focus and assessment mode were associated with the various components (optimization goal, alternative search, decision difficulty) of chronic maximizing outlined in the literature. We hypothesized that both promotion and assessment would positively predict possessing an optimization goal, but that only assessment, not promotion focus, would positively predict alternative search. We further hypothesized that assessment, but not promotion focus, 
would therefore positively predict chronic decision difficulty, one potential aggregate measure of the affective costs associated with decision-making.

As discussed above, we compared promotion focus to assessment mode because these are two motivational orientations that theoretically should share the optimization goal but diverge on alternative search. Thus, they are ideal candidates for our investigation into antecedents of relatively more and less adaptive maximizing. It should be noted that promotion focus and assessment mode are both part of broader motivational theories (regulatory focus and regulatory mode, respectively), and these theories include other distinct, orthogonal motivational constructs. Regulatory focus theory also posits prevention focus, which is concerned with security, safety, and vigilance (Higgins, 1997). Regulatory mode theory also posits locomotion, which is concerned with (psychological) movement from state to state (Higgins et al., 2003). However, neither prevention focus nor locomotion mode are theoretically linked to the desire for optimization, a critical prerequisite for identifying candidate antecedent factors for maximizing. Thus, these constructs are not the focus of the current investigation. ${ }^{1}$

\section{Method}

We recruited 681 participants $\left(48.3 \%\right.$ women; $\left.M_{\mathrm{age}}=33.49, S D=12.10\right)$ from Amazon Mechanical Turk as part of two larger studies. Sample size was determined in advance, and we aimed for approximately 350 participants for each study. This would provide $80 \%$ power to detect effects of approximately $r=.15$ ( $r=.11$ for the combined sample). All measures relevant for

\footnotetext{
${ }^{1}$ Although not the focus of our research, prevention focus and locomotion were measured in each of the studies as part of including the complete scales (Regulatory Focus Questionnaire and Regulatory Mode Scale). The results for promotion focus do not change when controlling for prevention focus, nor do the results for assessment change when controlling for locomotion. These analyses can be found in the Supplementary Online Materials (SOM).
} 
the current research occurred at the very beginning of the studies, before any other measures or manipulations. $^{2}$

Participants completed four self-report questionnaires. First, participants were given the Regulatory Focus Questionnaire (RFQ; Higgins et al., 2001; promotion focus, $\alpha=.69$ ), with six items assessing promotion focus on a 1-5 scale from "never or seldom" to "very often" (e.g., "I feel like I have made progress toward being successful in my life"). Next was the Regulatory Mode Scale (RMS; Kruglanski et al., 2000; assessment, $\alpha=.80$ ), with 12 items evaluating assessment on a 1-6 scale from "strongly disagree" to "strongly agree" (e.g., "I spend a great deal of time taking inventory of my positive and negative characteristics"). Third, we included the original Maximization Scale (MS; Schwartz et al., 2002; high standards, $\alpha=.68$; alternative search, $\alpha=.63$; decision difficulty, $\alpha=.66$ ), with 13 items (three for high standards, six for alternative search, and four for decision difficulty) on a 1-7 scale from "strongly disagree" to “strongly agree" (e.g., "No matter what I do, I have the highest standards for myself”). Finally, participants completed the Maximizing Tendency Scale (MTS; Diab et al., 2008; $\alpha=.85$ ), with 9 items on a 1-7 scale from "strongly disagree" to "strongly agree" (e.g., "I don't like having to settle for 'good enough'"). The MTS has shown similar patterns to the high standards subscale of the MS in previous research (Giacopelli et al., 2013; Rim et al., 2011; Weinhardt et al., 2012); thus, we included this scale to ensure that promotion and assessment were both positively correlated with high standards, using a scale that has arguably better psychometric properties relative to the original scale (Diab et al., 2008). ${ }^{3}$

\footnotetext{
${ }^{2}$ Which study participants were in did not moderate any of the results; we include it as a covariate in the following analyses.

${ }^{3}$ The MS and MTS include three duplicate items. A detailed comparison of these duplicated items is available in the SOM. We also provide analyses comparing our results when using revised versions of the MS and MTS as introduced by Nenkov et al. (2008) and Weinhardt et al. (2012).
} 


\section{Results}

Consistent with the previous research, the MTS was highly correlated with high standards, $r(651)=.80, p<.001$, and showed a moderate correlation with alternative search, $r(651)=.28, p<.001$. It was not correlated with decision difficulty, $r(651)=.02, p=.63$.

We regressed each component of maximizing and the MTS separately onto promotion and assessment. Using the overall maximizing score from the MS, it would appear that promotion focus is negatively associated and assessment positively associated with maximizing. However, as Table 1 shows, deconstructing this scale into its separate components of high standards, alternative search, and decision difficulty provides greater insight into how these motivational constructs relate to maximizing. As predicted, both promotion and assessment showed a positive association with high standards. However, also as predicted, only assessment showed positive associations with alternative search and decision difficulty, while promotion showed a slight negative association with alternative search and a strong negative association with decision difficulty. The MTS showed similar patterns as high standards, as expected.

\section{Discussion}

Study 1 provides initial evidence that promotion focus and assessment are associated with and differentially predict goals and strategies associated with maximizing. Thus, Study 1 provides evidence that promotion and assessment motivations could help differentiate between different types of maximizing, one positively associated with alternative search and decision difficulty, and the other negatively associated with these factors. In addition, our data are consistent with previous research suggesting that the strategy of searching through alternatives may be associated with negative decision experiences, at least at the chronic level (see also 
Nenkov et al., 2008; Purvis et al., 2011; Rim et al., 2011). Thus, Study 2 probes this question in more detail, moving from the chronic level to examining affective costs at the situational level.

\section{Study 2}

The primary goal of Study 2 was to examine whether the use of alternative search strategies would be associated with greater affective costs at the situational level. To do so, we

measured this strategy as a mediator of the influence of promotion and assessment on frustration with a discrete choice task. We wanted to assess whether alternative search would mediate the links between promotion and low frustration, and between assessment and high frustration. In particular, we wanted to compare alternative search as a mediator with the optimization goal as a mediator. In other words, we wanted to pit these two components against each other to determine whether it is the goals maximizers have (wanting the best) or the strategies they use (searching for alternatives) that are associated with higher or lower frustration when making a choice.

\section{Method}

We recruited 108 undergraduate participants $\left(67.3 \%\right.$ women; $\left.M_{\text {age }}=21.38, S D=3.94\right)$ from a mid-sized Canadian university to take part in an online study. Sample size was determined in advance, and was limited to the size of the participant pool available to us. This sample size provides $80 \%$ power to detect effects of approximately $r=.27$.

Participants first completed the same four questionnaires as in Study 1: the RFQ (promotion focus, $\alpha=.60$ ), RMS (assessment, $\alpha=.78$ ), MS (high standards, $\alpha=.61$; alternative search, $\alpha=.63$; decision difficulty, $\alpha=.69)$, and $\operatorname{MTS}(\alpha=.85)$.

After completing these scales, participants were given a hypothetical product choice task. They were asked to imagine that they were looking to purchase a vehicle, and were told to look at the available information and decide which one they would choose to buy for themselves. We 
chose vehicles as the product for this decision as vehicles offer numerous dimensions on which to evaluate, and constitute a relatively important decision for consumers. At the same time, it is a fairly prevalent consumer decision. Following the instructions, participants were given a table with either 5 or 50 vehicles, ${ }^{4}$ along with a number of characteristics of each car (e.g., price, fuel efficiency). Participants could take as long as they wanted to make their decision.

After they chose the car they preferred, participants were asked on a 1-7 scale how frustrated they felt when making the choice. ${ }^{5}$

\section{Results}

Motivational predictors. We first wanted to replicate the results from Study 1 on this university sample to ensure that the results were robust across populations. We regressed each component of maximizing and the MTS separately onto promotion and assessment.

As Table 1 shows, results were largely consistent with Study 1, with both promotion and assessment showing a positive association with high standards. However, only assessment showed positive associations with alternative search and decision difficulty, while promotion showed no association with alternative search and a negative association with decision difficulty.

\footnotetext{
${ }^{4}$ This study was originally designed to also test whether maximizers (versus non-maximizers) would be more overwhelmed or frustrated by large choice sets. Although we found an association between decision difficulty and frustration, $\beta=.30, t(99)=2.90, p=.01$, we did not find any main effect of the set size condition, or any interactions between condition and maximization. Condition also did not moderate the results of our mediation analyses. This is consistent with a meta-analysis suggesting no evidence for the choice overload effect (Scheibehenne, Greifeneder, \& Todd, 2010). Thus, the results presented here collapse across condition.

${ }^{5}$ Following Iyengar and Lepper (2000), we also had items about how much they enjoyed making the choice and how difficult it was. Although we find similar patterns among these three variables (frustration, reverse-coded enjoyment, and difficulty), frustration emerged as the strongest variable. Conceptually, frustration has the strongest link with negative affect, whereas one can experience even a difficult task as engaging or enjoyable. Thus, we focus our analyses on frustration. We also included several other items intended as procedural checks to make possible adjustments for future studies. This included items about satisfaction with the choice, feeling as though the decision was well-informed, and comprehensiveness of the options. In addition, we had some exploratory items about the strategies used to make the decision. We had no specific hypotheses about any of these items and are happy to provide additional details upon request.
} 
Alternative search as a mediator. We next examined the association between promotion and assessment on task frustration, to determine the mediating factors underlying this association. We predicted that promotion should lead to lower frustration, and assessment should lead to higher frustration. We also predicted that these associations would be mediated by alternative search, but not by high standards.

Using the PROCESS macro by Hayes (2013), we used bias-corrected bootstrapping with 5000 samples to examine the indirect effects of these two mediators. We included both high standards and alternative search as mediators in the model at the same time, and ran two separate models, one with promotion predicting task frustration (controlling for assessment), and another with assessment predicting task frustration (controlling for promotion).

Panels A and B in Figure 1 show that high standards did not show a significant indirect effect for either promotion or assessment. As predicted, for assessment, there was a significant indirect effect through alternative search to task frustration. This is consistent with the conclusion that individuals high in assessment experienced greater frustration as a result of greater use of alternative search strategies, rather than as a result of having higher standards. In contrast, alternative search did not show a significant indirect effect for promotion focus.

\section{Discussion}

Study 2 provides two key pieces of evidence that clarify the nature of maximizing. First, we replicated our results from Study 1 on a sample with very different demographic characteristics. This provides strong evidence for the robustness of the relationships between promotion and assessment with the goals and strategies associated with maximizing.

We also show evidence suggesting that alternative search strategies may be a critical mediating ingredient associated with affective costs of decision-making, particularly for 
assessment-focused individuals. We found that only alternative search strategies (and not the optimization goal) helped to explain why assessment was associated with higher task frustration. Putting maximizing goals and strategies in direct competition with each other, our mediation analyses are consistent with the interpretation that it is the type of maximizing strategies individuals use, not the maximizing goals they pursue, that are associated with affective costs on actual decision tasks. To the extent that individuals high in assessment make greater use of alternative search strategies, they are more likely to experience frustration when making decisions.

\section{Study 3}

The results of Study 2 suggest that alternative search is a strategy some maximizers use that is associated with affective costs on an actual decision task. In Study 3, we wanted to clarify the way in which the alternative search strategy manifests itself in a discrete decision task, as a way of understanding the behaviors that make this strategy maladaptive.

In this study, we compared exhaustive search (i.e., examination of multiple options), examination of options on multiple dimensions, time spent evaluating options, and returning to re-evaluate options that were previously eliminated (reconsideration). Very little research on maximizing to date has examined how maximizing manifests in discrete decision tasks, so our understanding of which behaviors might best reflect the chronic tendency to engage in alternative search is extremely limited. Thus, we made tentative predictions that alternative search would be associated with all of these behaviors. Of these behaviors, however, we predicted that reconsideration of options would be uniquely associated with affective costs on an actual decision task. 
As in Study 2, for Study 3 we aimed to investigate the behaviors associated with alternative search on a discrete choice task. These behaviors were measured with self-report items after the task, as well as recording of actual behavior during the task. In addition, we wanted to examine how these behaviors might predict affective costs on the task. In addition to frustration, in Study 3 we also measured post-choice regret, an affective experience that has been strongly linked with maximizing — and alternative search in particular — in past research (e.g., Diab et al., 2008; Iyengar et al., 2006; Nenkov et al., 2008; Purvis et al., 2011; Rim et al., 2011; Schwartz et al., 2002; Turner et al., 2012; Weinhardt et al., 2012). In the maximizing literature, both alternative search and regret have primarily been studied at the chronic, individual difference level. We attempted to provide evidence for these strategies and outcomes on a discrete decision task.

Finally, in Study 3 we wanted to present evidence for our overall model, from broad motivational orientations, to decision-making styles, to behaviors on a decision task, to affective costs. We first replicate the results from Study 2, testing high standards and alternative search as competing mechanisms explaining affective costs. Then, we test sequential mediation models to provide evidence for reconsideration of options as a mechanism for why alternative search is associated with affective costs.

\section{Method}

We recruited 210 undergraduate participants $\left(67.8 \%\right.$ women; $\left.M_{\text {age }}=20.29, S D=3.84\right)$ from a mid-sized Canadian university. Sample size was determined in advance, and was limited to the size of the participant pool available to us. This sample size provides $80 \%$ power to detect effects of approximately $r=.19$. Participants first completed the same questionnaires as in the previous 
studies: the RFQ (promotion focus, $\alpha=.62$ ), RMS (assessment, $\alpha=.70$ ), and MS (high standards, $\alpha=.73$; alternative search, $\alpha=.54$; decision difficulty, $\alpha=.58){ }^{6}$

After completing these scales, participants were given a product choice task very similar to Study 2. They were asked to imagine that they were looking to purchase a vehicle, and were told to review the available information and decide which one they would choose to buy for themselves. Following this, all participants were given a table with 20 vehicles, along with a number of characteristics of each car (e.g., price, fuel efficiency). Participants could take as long as they wanted to make their decision, and we recorded how long they spent on the choice task. A screenshot of what participants saw in the decision task is provided in the SOM.

After they chose the car they preferred, participants responded on a 1-7 scale to several items measuring self-reported behaviors on the task: four items $(\alpha=.74)$ regarding whether they reconsidered options after eliminating them (e.g., "I reconsidered an option that I had previously dismissed"), five items ( $\alpha=.81)$ about the regret they experienced (e.g., "I regret that I had to pass up other good options"), and three items $(\alpha=.86)$ about the extent to which they based their decision solely on the price of the option (e.g., "If an option was outside my price range, I didn't even look at it further"). They were also asked how frustrated they felt when making the choice on a $1-7$ scale. $^{7}$

Participants then responded to 10 items about the extent to which they engaged in exhaustive search (e.g., "I didn't eliminate any options until the very end of the process"). An

\footnotetext{
${ }^{6}$ In Study 3, we also included the MTS (Diab et al., 2008) and Maximization Inventory (Turner et al., 2012) along with the MS. These scales show very similar patterns to their corresponding MS subscales. For consistency with Studies 1-2, we present the MS results here; analyses using the MTS and MI are available in the SOM.

${ }^{7}$ As in Study 2, we also had items about how much they enjoyed making the choice and how difficult it was, which showed similar patterns to frustration.
} 
exploratory factor analysis of this scale showed two factors, corresponding to the extent to which they examined dimensions $(\alpha=.71)$, and the extent to which they examined options $(\alpha=.67) .^{8}$

In addition to our self-reported behaviors, we set up the decision task itself in a way that could capture actual behavioral data. Beside each row and column were checkboxes that participants could use to show or hide the information in that row or column. All the information was visible by default, but participants could use these checkboxes to eliminate information from the screen (or bring it back, if they chose to do so). We suggested in the instructions that these checkboxes could be used after eliminating an option or characteristic from consideration (see the Appendix for instruction wording). Given the way the information was displayed, hiding a row was indicative of removing an option from one's choice set. Hiding a column, on the other hand, was indicative of removing a dimension upon which to evaluate options (e.g., price, fuel efficiency). In essence, hiding columns reduced the complexity of the choice, while hiding rows reduced the size of the choice set.

During this task, the computer was programmed to keep track of how long participants spent on the task, how many rows and columns they unchecked to hide, as well as how many rows and columns they selected to be displayed again, if any.

\section{Results}

The zero-order correlations of each dependent variable with the MS subscales are displayed in Table 2. For each of our dependent variables we also examined a regression model including all three MS subscales as predictors, also displayed in Table 2. Given that there are small but significant correlations between the three subscales ( $r$ s from .16 to $.27, p s<.02$ ), both the zero-order correlations and the regression models are instructive.

\footnotetext{
${ }^{8}$ More details about this factor analysis can be found in the SOM.
} 
Self-reported information selection. Following the task, we asked participants about the extent to which they engaged in exhaustive search, including measures of their evaluation of dimensions (columns) and their evaluation of options (rows).

Interestingly, alternative search was not associated with greater self-reported evaluation of dimensions. High standards was positively associated with more evaluation of dimensions. In addition, decision difficulty, when included in a model with high standards and alternative search, was marginally associated with reduced evaluation of dimensions.

With regard to evaluating options, high standards, alternative search, and decision difficulty were all associated with more evaluation of options at the zero-order level. When included in the model together, however, none of these relationships emerged as significant.

Self-reported reconsideration. We asked participants to report how much they reconsidered options they had previously eliminated from consideration. At the zero-order level, both alternative search and decision difficulty were significantly associated with greater reported reconsideration of options. However, as simultaneous predictors, only decision difficulty was significantly associated with greater reconsideration of options.

Self-reported reconsideration was strongly correlated with regret, $r(197)=.61, p<.001$, which we analyze below. It was also correlated with the behavioral measure of the number of rows reselected, $r(197)=.18, p=.01$. However, it was not associated with the number of columns reselected, $r(197)=.07, p=.30$.

Behavioral measures. Alternative search significantly predicted hiding fewer columns, both at the zero-order level and when included simultaneously with the other subscales. This may suggest one behavioral cue with which alternative search is associated: removing fewer dimensions from consideration when engaging in a choice task. 
However, interpretation of some of our other behavioral measures was made difficult because some of the key behaviors were quite infrequent. For example, as our measure of reconsidering options or dimensions, we examined how many times participants selected a previously hidden column or row to make it visible again. On the whole, this was uncommon behavior: participants reselected on average .57 columns $(S D=1.02)$ and 1.16 rows $(S D=2.43)$. As such, none of the maximizing subscales predicted the other behavioral items, including time spent on the task.

Post-decision emotions. Two key emotional outcomes were assessed with regard to the choice task: frustration and regret. High standards did not predict frustration or regret; however, alternative search and decision difficulty were both significantly associated with greater frustration and greater regret. This was true for both the zero-order correlations and when entered as simultaneous predictors.

Promotion and assessment. Our theoretical model conceptualizes promotion and assessment as distal factors primarily influencing decision experiences through maximizing processes. However, as shown in Table 2, regression analyses did reveal some direct associations between these motivational orientations and our dependent variables. In particular, assessment marginally predicted hiding fewer columns, and both promotion and assessment were associated with greater self-reported evaluation of dimensions. In addition, assessment predicted greater self-reported reconsideration, and promotion marginally predicted less frustration on the task. Promotion also predicted less regret, while assessment predicted greater regret on the task.

Replicating Study 2. We again used bias-corrected bootstrapping with 5000 samples to examine the indirect effects of high standards and alternative search, as shown in Figure 1. We used these mediation models to predict task frustration and regret. 
When predicting frustration, we found that there was no indirect effect through high standards either for promotion focus, $B=.01,95 \%$ CI $[-.12, .14]$, or for assessment mode, $B=.01$, 95\% CI $[-.13, .15]$. There was also no indirect effect through alternative search for promotion focus, $B=-.06,95 \% \mathrm{CI}[-.21, .02]$; however, alternative search significantly mediated the relationship between assessment and frustration, $B=.13,95 \%$ CI $[.04, .28]$.

We found similar results when predicting regret. There was no indirect effect through high standards either for promotion focus, $B=.003,95 \%$ CI $[-.09, .09]$, or for assessment mode, $B=.003,95 \%$ CI $[-.09, .10]$. There was also no indirect effect through alternative search for promotion focus, $B=-.03,95 \% \mathrm{CI}[-.13, .01]$; however, alternative search significantly mediated the relationship between assessment and regret, $B=.08,95 \%$ CI $[.01, .19]$.

Sequential mediation path analysis. In order to test our complete model of the process from motivational orientations to affective costs, we developed a path model using Amos 20. Unfortunately, the behavioral measures we captured in this study did not correlate strongly with frustration and regret (with correlations ranging from -.08 to .10, and $p \mathrm{~s}>.16$ ). This made them unlikely candidates for mediation between alternative search and decision outcomes. However, the self-reported evaluation of options was correlated with frustration, $r(198)=.27, p<.001$, and with regret, $r(197)=.30, p<.001$. Self-reported reconsideration was also correlated with frustration, $r(196)=.47, p<.001$, and regret, $r(197)=.61, p<.001$. Thus, we used these two variables in our model to determine the extent to which they mediated the relationships between alternative search and frustration or regret.

Using these variables, we created a dual sequential mediation model (see Figure 2) where promotion focus and assessment mode each predicted high standards and alternative search, which in turn each predicted evaluation of options and reconsideration of options. These two 
self-report measures then each predicted frustration and regret. Both variables at each stage in the model were allowed to covary, and we included all direct effects to create a fully saturated model. We were particularly interested in the indirect pathways from promotion and assessment through alternative search, then testing evaluation of options and reconsideration of options as competing mediators predicting the outcome measures. We also tested the mediation pathways going through high standards. We used 5000 bias-corrected bootstrapped samples to estimate the confidence intervals of the indirect effects. The complete model is shown in Figure 2, and the key direct and indirect effects are shown in Table 3.

As shown in Table 3, promotion focus did not have any significant indirect effects predicting frustration or regret. The associations between assessment mode predicting frustration and regret, however, was mediated through the sequential mediators of alternative search and reconsideration of options. Evaluation of options did not mediate these associations. This suggests that it was reconsideration of options (and not evaluation of options) that led assessment-focused individuals who chronically engage in alternative search to experience higher frustration on the task and greater post-decision regret.

\section{Discussion}

Study 3 provided greater insight on several key points. First, it showed several decision behaviors with which alternative search is associated. People high in alternative search were less likely to remove dimensions from evaluation, and reported engaging in both greater evaluation and greater reconsideration of options. As hypothesized, however, the element of alternative search that was problematic was not more exhaustive evaluation itself, but the increased likelihood of reconsidering options. When examining evaluation and reconsideration of options as simultaneous mediators of the links between alternative search and frustration/regret in our 
mediation model, the results were consistent with the interpretation that reconsideration of options, and not simply greater evaluation of options, explained these associations.

Some research has suggested that the regret felt by maximizers may be fuelled by retrospectively thinking about forgone opportunities (Huang \& Zeelenberg, 2012; Iyengar et al., 2006). The present research suggests that, for individuals high in alternative search, the seeds of this regret may be formed during the decision process itself as a result of continuing to consider (and reconsider) previously dismissed options rather than dismissing them permanently. In contrast, individuals with high standards were able to perform these decision tasks without greater frustration or regret; this suggests once again that it is not the goal of "wanting the best option" that is responsible for affective costs, but rather the strategies that individuals are using — and the behaviors these strategies produce — to pursue this maximizing goal.

Second, this study showed support for our overall model, where both promotion focus and assessment mode predicted high standards, but only assessment mode predicted alternative search. The positive association with alternative search, in turn, was linked with downstream affective costs on an actual decision task. When combined with Studies 1 and 2, this offers further support for how broad, chronic motivational orientations are associated with chronic decision-making styles, which in turn influence experiences on a concrete decision. This study thus offers a glimpse at how these chronic motivational and maximizing individual differences are linked with specific behaviors on a discrete decision task, something which has received limited attention in the literature to date (though see Dalal et al., 2015; Iyengar et al., 2006; Polman, 2010; Rim et al., 2011). 


\section{General Discussion}

Across three studies, we have shown evidence that distinguishing between the goals and strategies of maximizers can predict the affective costs that maximizers experience. We proposed that pursuing the "best" option could be understood in terms of an optimization goal, while alternative search was a strategy that maximizers may use during decision tasks. However, we showed that decision difficulty, frustration, and regret are by no means inevitable experiences for maximizers; indeed, Studies 2 and 3 demonstrate that it is the alternative search strategy, not the optimization goal, that is linked with affective costs when making decisions. Study 3 provides evidence for one element of an alternative search strategy—reconsideration of options, not exhaustive evaluation - that is particularly maladaptive.

We also provided evidence in all three studies for two antecedent motivational orientations that lead to maximizing: promotion focus and assessment mode. Both of these orientations are associated with an optimization goal, but only assessment is positively associated with alternative search. By differentiating between the goals and strategies of decision-makers, we can better understand how the underlying motivations individuals have can lead them to maximize or not, as well as what strategies they use to engage the decision process.

One important contribution of this work is to distinguish between different types or profiles of maximizers. Previous research on maximizing — especially as measured by the Maximization Scale - may have indeed been studying one relatively common and coherent type of maximizer (Schwartz et al., 2002). However, other researchers (e.g., Diab et al., 2008; Nenkov et al., 2008) have been right to point out that disentangling the optimization goal from alternative search may reveal more adaptive types of maximizing. What we have added to this discussion is to show how underlying motivations can serve to bind goals and strategies together 
to create coherent profiles of maximizers. For example, assessment mode serves to bind an optimization goal with a particular strategy of alternative search, which then results in negative experiences when making decisions (chronic decision difficulty, frustration, regret). We have not yet identified a particular strategy that individuals high in promotion focus use to pursue their optimization goal. However, one intriguing possibility from Dalal et al. (2015) is that promotionfocused individuals could engage in greater "strategic search." These researchers found that high standards (and the MTS scale), but not alternative search, was correlated with searching through information about options more systematically (either comparing options one attribute at a time, or comparing attributes one option at a time). Although Dalal et al. did not measure promotion focus, strategic search is one possible strategy for future research to examine. In addition, our research here was correlational in nature; as such, it would also be helpful to experimentally manipulate some of these motivational orientations to verify the causal pathway from motivations to maximizing.

Another important contribution of the current research is to examine maximizing in the context of discrete decision tasks (Studies 2 and 3). To date, such methodology has been relatively rare in the maximizing literature, and as a result, little insight has been gleaned as to the real-time processes that occur during the decisions of maximizers. These processes and immediate affective experiences at the situational level may have important consequences for the broad, chronic negative experiences studied in the literature to date. For example, indecision regarding future career plans in adolescents and undergraduates has been associated with lower self-efficacy, life satisfaction, and self-esteem, and greater depression (Creed, Prideaux, \& Patton, 2005; Saunders, Peterson, Sampson Jr., \& Reardon, 2000). Regrets over specific goals and events has also been associated with lower life satisfaction and greater repetitive thoughts 
across the lifespan (Epstude \& Jonas, 2014; Jokisaari, 2003). On this basis, we argue that if individuals are more likely to experience affective costs in any given decision, these may aggregate over time leading to broader negative experiences. However, future work should implement a longitudinal approach to strengthen this link between the situational and dispositional levels. In addition, as the decisions in our research were not consequential for participants, we also recognize the continued importance of conducting future work examining consequential decision tasks, as well as decisions in domains other than consumer goods.

\section{Future Directions}

Much work remains to be done to integrate the past research on maximizing. With numerous scales and a variety of definitions, understanding exactly what maximizing predicts and is predicted by remains a challenge. Our motivational framework, differentiating between goals and strategies, can help to provide direction to how such integration can continue; our hope is that if researchers can agree on how to conceptualize maximization, it will provide common ground from which to work.

We believe that this motivational framework may also provide insights into how to conceptualize satisficing, a construct that some have argued to be orthogonal to maximizing (Turner et al., 2012; Weinhardt et al., 2012). Satisficing has typically been described as involving both the goal of finding a "good enough" option and the strategy of less intensive search. However, we propose that, as we have argued with maximizing, it is possible to differentiate between the goals and strategies of satisficers. Thus, maximizers and satisficers have different goals ("best" versus "good enough"), but their strategies could potentially overlap. Without this differentiation, whether satisficing is orthogonal or not could be a function of how it 
was measured. In addition, as with maximizing, there may be more or less adaptive forms of satisficing revealed by the strategies people use.

The framework we have described also highlights areas in which work remains to be done. First, further research is needed to find other antecedent motivational (and other) factors associated with the development, manifestation, and maintenance of maximizing tendencies. There are undoubtedly other constructs as well that may shed light on how maximizing operates. Such research will greatly assist in the understanding of what underlies maximizing, and may also provide insight into how to help maximizers with a maladaptive profile to use more adaptive strategies.

Second, it is important to develop better situational measures of maximizing behavior in actual decisions to supplement the existing research of maximizing at the chronic (dispositional) level. This will not only allow researchers to discover when and how maximizing occurs in a particular situation, but also might help to clarify differences between the existing dispositional scales. Study 3 began to investigate different behavioral components of alternative search, but future research is needed to understand how different chronic measures of alternative search predict behaviors within situations. The answer to these questions may be illuminated with better behavioral or situational measures of maximizing.

Third, there is still ample room for research into the various strategies that maximizers use when making decisions. Up to this point, research has primarily focused on alternative search strategies, and indeed many researchers have defined them as central to the concept of maximizing (Lai, 2010; Nenkov et al., 2008; Schwartz et al., 2002; Turner et al., 2012). Being able to identify other strategies, as well as how dispositional and situational variables might predict which strategies are used, would be invaluable to better understand how maximizing 
manifests. It would also be valuable to investigate how these strategies may lead to more or less optimal decisions in terms of the actual utility provided by the chosen option. Being able to investigate not only the immediate affective costs of different strategies, but also their downstream consequences for decision satisfaction, would be a profitable avenue for future research.

We are hopeful that the framework we have advanced here will assist in resolving some of the issues that have complicated past research on maximizing, as well as guide future research in the area. There are still many questions left to be answered, but we are confident that with the right goals (finding the best answers) and the right strategies (using a unified framework), we can discover the answers without difficulty. 


\section{References}

Arkes, H. R., \& Blumer, C. (1985). The psychology of sunk cost. Organizational Behavior and Human Decision Processes, 35, 124-140. http://doi.org/10.1016/0749-5978(85)90049-4

Avnet, T., \& Higgins, E. T. (2003). Locomotion, assessment, and regulatory fit: Value transfer from "how" to "what." Journal of Experimental Social Psychology, 39(5), 525-530. http://doi.org/10.1016/S0022-1031(03)00027-1

Benjamin, L., \& Flynn, F. J. (2006). Leadership style and regulatory mode: Value from fit? Organizational Behavior and Human Decision Processes, 100(2), 216-230. http://doi.org/10.1016/j.obhdp.2006.01.008

Creed, P., Prideaux, L.-A., \& Patton, W. (2005). Antecedents and consequences of career decisional states in adolescence. Journal of Vocational Behavior, 67(3), 397-412. http://doi.org/10.1016/j.jvb.2004.08.008

Crowe, E., \& Higgins, E. T. (1997). Regulatory focus and strategic inclinations: Promotion and prevention in decision-making. Organizational Behavior and Human Decision Processes, 69(2), 117-132. http://doi.org/10.1006/obhd.1996.2675

Dalal, D. K., Diab, D. L., Zhu, X., \& Hwang, T. (2015). Understanding the construct of maximizing tendency: A theoretical and empirical evaluation. Journal of Behavioral Decision Making, 28, 437-450. http://doi.org/10.1002/bdm.1859

Dar-Nimrod, I., Rawn, C. D., Lehman, D. R., \& Schwartz, B. (2009). The maximization paradox: The costs of seeking alternatives. Personality and Individual Differences, 46, 631635. http://doi.org/10.1016/j.paid.2009.01.007

Diab, D. L., Gillespie, M. A., \& Highhouse, S. (2008). Are maximizers really unhappy? The measurement of maximizing tendency. Judgment and Decision Making, 3(5), 364-370. 
Epstude, K., \& Jonas, K. J. (2014). Regret and counterfactual thinking in the face of inevitability: The case of HIV-positive men. Social Psychological and Personality Science, 6(2), 157163. http://doi.org/10.1177/1948550614546048

Förster, J., \& Higgins, E. T. (2005). How global versus local perception fits regulatory focus. Psychological Science, 16(8), 631-636. http://doi.org/10.1111/j.1467-9280.2005.01586.x

Förster, J., Higgins, E. T., \& Bianco, A. T. (2003). Speed/accuracy decisions in task performance: Built-in trade-off or separate strategic concerns? Organizational Behavior and Human Decision Processes, 90(1), 148-164. http://doi.org/10.1016/S0749-5978(02)005095

Frost, R. O., \& Shows, D. L. (1993). The nature and measurement of compulsive indecisiveness. Behaviour Research and Therapy, 31(7), 683-692. http://doi.org/10.1016/00057967(93)90121-A

Fuglestad, P. T., Rothman, A. J., \& Jeffery, R. W. (2008). Getting there and hanging on: The effect of regulatory focus on performance in smoking and weight loss interventions. Health Psychology, 27(3(Suppl.)), S260-S270. http://doi.org/10.1037/0278-

6133.27.3(Suppl.).S260

Giacopelli, N. M., Simpson, K. M., Dalal, R. S., Randolph, K. L., \& Holland, S. J. (2013). Maximizing as a predictor of job satisfaction and performance: A tale of three scales. Judgment and Decision Making, 8(4), 448-469.

Grant, H., \& Higgins, E. T. (2003). Optimism, promotion pride, and prevention pride as predictors of quality of life. Personality and Social Psychology Bulletin, 29(12), 15211532. http://doi.org/10.1177/0146167203256919

Hayes, A. F. (2013). Introduction to mediation, moderation, and conditional process analysis: A 
regression-based approach. New York: Guilford Press.

Higgins, E. T. (1997). Beyond pleasure and pain. American Psychologist, 52(12), 1280-1300. http://doi.org/10.1037//0003-066X.52.12.1280

Higgins, E. T. (2011). What is motivation? In Beyond pleasure and pain: How motivation works (pp. 17-66). New York: Oxford University Press.

Higgins, E. T., Friedman, R. S., Harlow, R. E., Idson, L. C., Ayduk, O. N., \& Taylor, A. (2001). Achievement orientations from subjective histories of success: Promotion pride versus prevention pride. European Journal of Social Psychology, 31, 3-23. http://doi.org/10.1002/ejsp.27

Higgins, E. T., Kruglanski, A. W., \& Pierro, A. (2003). Regulatory mode: Locomotion and assessment as distinct orientations. Advances in Experimental Social Psychology, 35, 293344. http://doi.org/10.1016/S0065-2601(03)01005-0

Hsee, C. K., \& Hastie, R. (2006). Decision and experience: Why don't we choose what makes us happy? Trends in Cognitive Sciences, 10(1), 31-37. http://doi.org/10.1016/j.tics.2005.11.007

Huang, W.-H., \& Zeelenberg, M. (2012). Investor regret: The role of expectation in comparing what is to what might have been. Judgment and Decision Making, 7(4), 441-451.

Iyengar, S. S., \& Lepper, M. R. (2000). When choice is demotivating: Can one desire too much of a good thing? Journal of Personality and Social Psychology, 79(6), 995-1006. http://doi.org/10.1037/0022-3514.79.6.995

Iyengar, S. S., Wells, R. E., \& Schwartz, B. (2006). Doing better but feeling worse: Looking for the "best" job undermines satisfaction. Psychological Science, 17(2), 143-150. http://doi.org/10.1111/j.1467-9280.2006.01677.x 
Jokisaari, M. (2003). Regret appraisals, age, and subjective well-being. Journal of Research in Personality, 37(6), 487-503. http://doi.org/10.1016/S0092-6566(03)00033-3

Kruglanski, A. W., Shah, J. Y., Fishbach, A., Friedman, R., Chun, W. Y., \& Sleeth-Keppler, D. (2002). A theory of goal systems. Advances in Experimental Social Psychology, 34, 331378.

Kruglanski, A. W., Thompson, E. P., Higgins, E. T., Atash, M. N., Pierro, A., Shah, J. Y., \& Spiegel, S. (2000). To "do the right thing" or to "just do it": Locomotion and assessment as distinct self-regulatory imperatives. Journal of Personality and Social Psychology, 79(5), 793-815. http://doi.org/10.1037/0022-3514.79.5.793

Lai, L. (2010). Maximizing without difficulty: A modified maximizing scale and its correlates. Judgment and Decision Making, 5(3), 164-175.

Leder, S., Florack, A., \& Keller, J. (2014). Self-regulation and protective health behaviour: How regulatory focus and anticipated regret are related to vaccination decisions. Psychology \& Health, 30(2), 165-188. http://doi.org/10.1080/08870446.2014.954574

Liberman, N., Molden, D. C., Idson, L. C., \& Higgins, E. T. (2001). Promotion and prevention focus on alternative hypotheses: Implications for attributional functions. Journal of Personality and Social Psychology, 80(1), 5-18. http://doi.org/10.1037/0022-3514.80.1.5

Lin, C.-H., \& Huang, C.-W. (2011). The effects of regulatory focus and bundle-pricing framing on consumers' perceptions of loss. Social Behavior and Personality, 39(1), 113-118. http://doi.org/10.2224/sbp.2011.39.1.113

Mauro, R., Pierro, A., Mannetti, L., Higgins, E. T., \& Kruglanski, A. W. (2009). The perfect mix: Regulatory complementarity and the speed-accuracy balance in group performance. Psychological Science, 20(6), 681-685. http://doi.org/10.1111/j.1467-9280.2009.02363.x 
Nenkov, G. Y., Morrin, M., Ward, A., Schwartz, B., \& Hulland, J. (2008). A short form of the Maximization Scale: Factor structure, reliability and validity studies. Judgment and Decision Making, 3(5), 371-388.

Nolen-Hoeksema, S. (2000). The role of rumination in depressive disorders and mixed anxiety/depressive symptoms. Journal of Abnormal Psychology, 109(3), 504-511. http://doi.org/10.1037/0021-843X.109.3.504

Nolen-Hoeksema, S., Larson, J., \& Grayson, C. (1999). Explaining the gender difference in depressive symptoms. Journal of Personality and Social Psychology, 77(5), 1061-1072. http://doi.org/10.1037/0022-3514.77.5.1061

Pierro, A., Giacomantonio, M., Pica, G., Mannetti, L., Kruglanski, A. W., \& Higgins, E. T. (2013). When comparative ads are more effective: Fit with audience's regulatory mode. Journal of Economic Psychology, 38, 90-103. http://doi.org/10.1016/j.joep.2012.10.006

Polman, E. (2010). Why are maximizers less happy than satisficers? Because they maximize positive and negative outcomes. Journal of Behavioral Decision Making, 23, 179-190. http://doi.org/10.1002/bdm

Purvis, A., Howell, R. T., \& Iyer, R. (2011). Exploring the role of personality in the relationship between maximization and well-being. Personality and Individual Differences, 50(3), 370375. http://doi.org/10.1016/j.paid.2010.10.023

Ratner, R. K., Kahn, B. E., \& Kahneman, D. (2014). Choosing less-preferred experiences for the sake of variety. Journal of Consumer Research, 26(1), 1-15. http://doi.org/10.1086/209547

Rim, H. Bin, Turner, B. M., Betz, N. E., \& Nygren, T. E. (2011). Studies of the dimensionality, correlates, and meaning of measures of the maximizing tendency. Judgment and Decision Making, 6(6), 565-579. 
Rivis, A., Sheeran, P., \& Armitage, C. J. (2010). Explaining adolescents' cigarette smoking: A comparison of four modes of action control and test of the role of self-regulatory mode. Psychology \& Health, 25(8), 893-909. http://doi.org/10.1080/08870440902850310

Saunders, D. E., Peterson, G. W., Sampson Jr., J. P., \& Reardon, R. C. (2000). Relation of depression and dysfunctional career thinking to career indecision. Journal of Vocational Behavior, 56(2), 288-298. http://doi.org/10.1006/jvbe.1999.1715

Scheibehenne, B., Greifeneder, R., \& Todd, P. M. (2010). Can there ever be too many options? A meta-analytic review of choice overload. Journal of Consumer Research, 37(3), 409-425. http://doi.org/10.1086/651235

Scholer, A. A., \& Higgins, E. T. (2011). Promotion and prevention systems: Regulatory focus dynamics within self-regulatory hierarchies. In K. D. Vohs \& R. F. Baumeister (Eds.), Handbook of self-regulation: Research, theory, and applications (2nd ed., pp. 143-161). New York: Guilford Press.

Schwartz, B., Ward, A., Monterosso, J., Lyubomirsky, S., White, K., \& Lehman, D. R. (2002). Maximizing versus satisficing: Happiness is a matter of choice. Journal of Personality and Social Psychology, 83(5), 1178-1197. http://doi.org/10.1037//0022-3514.83.5.1178

Simon, H. A. (1955). A behavioral model of rational choice. Quarterly Journal of Economics, 59, 99-118. http://doi.org/10.2307/1884852

Simon, H. A. (1956). Rational choice and the structure of the environment. Psychological Review, 63(2), 129-138. http://doi.org/10.1037/h0042769

Stam, D. A., Van Knippenberg, D., \& Wisse, B. (2010). The role of regulatory fit in visionary leadership. Journal of Organizational Behavior, 31, 499-518. http://doi.org/10.1002/job.624 
Turner, B. M., Rim, H. Bin, Betz, N. E., \& Nygren, T. E. (2012). The Maximization Inventory. Judgment and Decision Making, 7(1), 48-60.

Weinhardt, J. M., Morse, B. J., Chimeli, J., \& Fisher, J. (2012). An item response theory and factor analytic examination of two prominent maximizing tendency scales. Judgment and Decision Making, 7(5), 644-658.

Zou, X., \& Scholer, A. A. (2016). Motivational affordance and risk-taking across decision domains. Personality and Social Psychology Bulletin, 42(3), 275-289. http://doi.org/10.1177/0146167215626706 
Table 1

Regressing maximizing components on motivational predictors (Studies 1 and 2)

\begin{tabular}{|c|c|c|c|c|c|}
\hline & $\underline{\text { MS }}$ & $\begin{array}{c}\underline{\text { High }} \\
\underline{\text { Standards }}\end{array}$ & $\frac{\text { Alternative }}{\underline{\text { Search }}}$ & $\frac{\text { Decision }}{\text { Difficulty }}$ & $\underline{\mathrm{MTS}}$ \\
\hline \multicolumn{6}{|l|}{ Study 1} \\
\hline Promotion & $\begin{array}{c}-.10 * * \\
{[-.18,-.03]}\end{array}$ & $\begin{array}{c}.38 * * * \\
{[.31, .45]}\end{array}$ & $\begin{array}{c}-.07^{+} \\
{[-.14, .01]}\end{array}$ & $\begin{array}{c}-.37 * * * \\
{[-.44,-.30]}\end{array}$ & $\begin{array}{c}.36 * * * \\
{[.29, .43]}\end{array}$ \\
\hline Assessment & $\begin{array}{c}.37 * * * \\
{[.30, .44]}\end{array}$ & $\begin{array}{c}.26 * * * \\
{[.19, .33]}\end{array}$ & $\begin{array}{c}.33 * * * \\
{[.25, .40]}\end{array}$ & $\begin{array}{c}.16 * * * \\
{[.09, .23]}\end{array}$ & $\begin{array}{c}.27 * * * \\
{[.20, .35]}\end{array}$ \\
\hline \multicolumn{6}{|l|}{ Study 2} \\
\hline Promotion & $\begin{array}{c}-.09 \\
{[-.28, .09]}\end{array}$ & $\begin{array}{c}.28 * * \\
{[.09, .47]}\end{array}$ & $\begin{array}{c}-.13 \\
{[-.32, .06]}\end{array}$ & $\begin{array}{c}-.21 * \\
{[-.40,-.02]}\end{array}$ & $\begin{array}{c}.18^{+} \\
{[-.01, .37]}\end{array}$ \\
\hline Assessment & $\begin{array}{c}.33^{* *} \\
{[.15, .52]}\end{array}$ & $\begin{array}{c}.22^{*} \\
{[.03, .41]}\end{array}$ & $\begin{array}{c}.28 * * \\
{[.10, .47]}\end{array}$ & $\begin{array}{c}.21 * \\
{[.03, .40]}\end{array}$ & $\begin{array}{c}.20 * \\
{[.01, .40]}\end{array}$ \\
\hline
\end{tabular}

Note. Values are standardized betas $(\beta)$. Confidence intervals $(\mathrm{CI})$ are $95 \%$ confidence intervals. ${ }^{+} p<.10 ; * p<.05 ; * * p<.01 ; * * * p<.001$ 
Table 2

Regressing task behaviors and outcomes on maximizing components (Study 3)

\begin{tabular}{|c|c|c|c|c|c|c|}
\hline & & elf-report it & $\mathbf{m s}$ & $\begin{array}{c}\text { Behavioral } \\
\text { items }\end{array}$ & Outcor & \\
\hline & $\begin{array}{c}\text { Evaluating } \\
\text { Dimensions }\end{array}$ & $\frac{\text { Evaluating }}{\text { Options }}$ & $\frac{\text { Reconsidering }}{\underline{\text { Options }}}$ & $\underline{\text { Cols. }}$ & $\underline{\text { Frustration }}$ & $\underline{\text { Regret }}$ \\
\hline \multicolumn{7}{|c|}{ Zero-order correlations } \\
\hline $\begin{array}{l}\text { MS-high } \\
\text { standards }\end{array}$ & .19 & $.13^{+}$ & & & & \\
\hline $\begin{array}{l}\text { MS-alt. } \\
\text { search }\end{array}$ & & .15 & .17 & -.15 & .23 & .20 \\
\hline $\begin{array}{l}\text { MS-decision } \\
\text { diff. }\end{array}$ & & .15 & .30 & & .27 & .32 \\
\hline Promotion & .15 & & & & $-.13^{+}$ & -.22 \\
\hline Assessment & $.13^{+}$ & & .16 & $-.13^{+}$ & & .15 \\
\hline \multicolumn{7}{|c|}{$\underline{\text { Regression models }}$} \\
\hline \multicolumn{7}{|c|}{ Model 1} \\
\hline $\begin{array}{l}\text { MS-high } \\
\text { standards }\end{array}$ & .20 & & & & & \\
\hline $\begin{array}{l}\text { MS-alt. } \\
\text { search }\end{array}$ & & & & -.15 & .18 & .14 \\
\hline $\begin{array}{l}\text { MS-decision } \\
\text { diff. }\end{array}$ & $-.12^{+}$ & & .27 & & .23 & .29 \\
\hline \multicolumn{7}{|l|}{ Model 2} \\
\hline Promotion & .17 & & & & $-.13^{+}$ & -.20 \\
\hline Assessment & .15 & & .15 & $-.13^{+}$ & & $.13^{+}$ \\
\hline \multicolumn{7}{|c|}{$\begin{array}{l}\text { Notes. Values for regression models are standardized betas }(\beta) \text {. Only significant and marginal } \\
(.05<p<.10) \text { associations are shown; marginal associations are indicated with a plus sign }\left(^{+}\right) \text {. } \\
\text { Some behavioral items - number of rows hidden, time on task, reselecting rows, and reselecting }\end{array}$} \\
\hline
\end{tabular}


Table 3

Direct and indirect effects of motivation and maximizing on task outcomes (Study 3)

\section{IV}

Direct effects

Promotion

Promotion

Assessment

Assessment

High standards

High standards

Alt. search

Alt. search

Evaluation of

options

Evaluation of

options

Reconsideration

Reconsideration $\underline{\text { Mediator } 1}$

$\underline{\text { Mediator } 2}$
$\underline{\mathrm{DV}}$

High standards

Alt. search

High standards

Alt. search

Evaluation of options

Reconsideration

Evaluation of options

Reconsideration

Frustration

Regret
Frustration
Regret

Indirect effects

Promotion

Promotion

Promotion

Promotion

Promotion

Promotion

Promotion

Promotion

Assessment

Assessment

Assessment

Assessment

Assessment

Assessment

Assessment

Assessment
High standards

High standards

High standards

High standards

Alt. search

Alt. search

Alt. search

Alt. search

High standards

High standards

High standards

High standards

Alt. search

Alt. search

Alt. search

Alt. search
Eval. options

Eval. options

Reconsideration

Reconsideration

Eval. options

Eval. options

Reconsideration

Reconsideration

Eval. options

Eval. options

Reconsideration

Reconsideration

Eval. options

Eval. options
Reconsideration
Frustration

Regret

Frustration

Regret

Frustration

Regret

Frustration

Regret

Frustration

Regret

Frustration

Regret

Frustration

Regret

Frustration

Regret $\underline{\text { Estimate }}$

$95 \% \mathrm{CI}$

Notes. Estimates are unstandardized effects. Confidence intervals (CI) are 95\% confidence intervals, and were estimated with 5000 bias-corrected bootstrapped samples. See Figure 2 for depiction of full model; ${ }^{+} p<.10 ; * p<.05 ; * * p<.01 ; * * * p<.001$ 


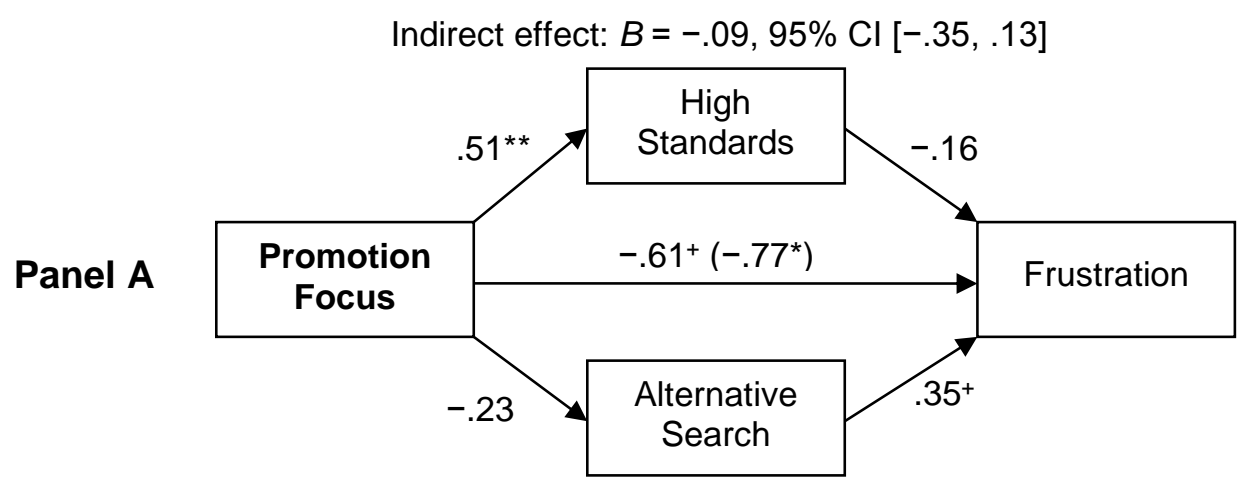

Indirect effect: $B=-.07,95 \% \mathrm{Cl}[-.34, .03]$

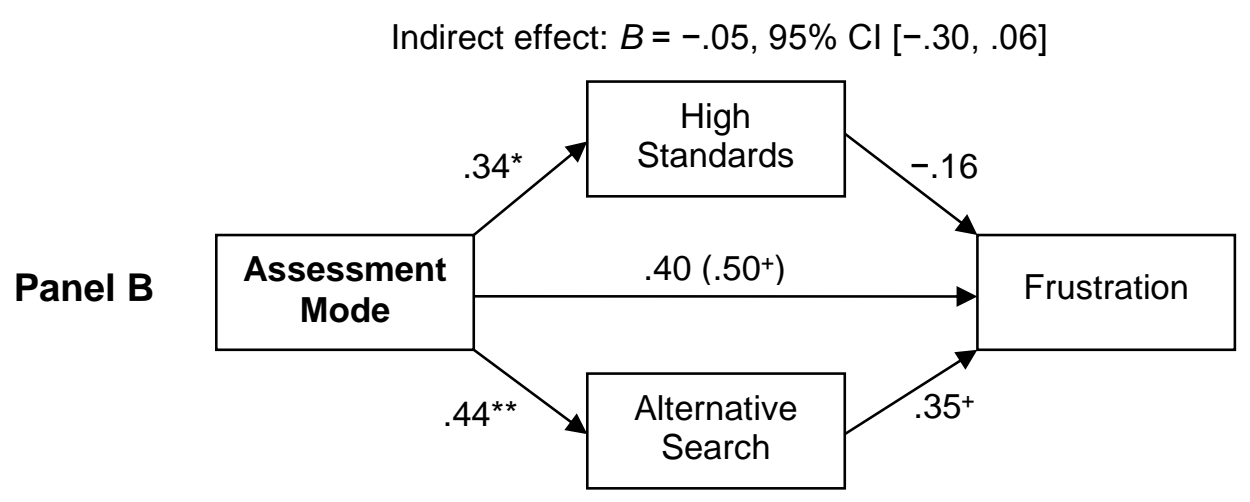

Indirect effect: $B=.16,95 \% \mathrm{Cl}[.02, .41]$

Figure 1. Concurrent mediation models for promotion focus (Panel A) and assessment mode (Panel B) predicting task frustration, mediated by high standards and alternative search (Study 2). The analyses for promotion focus controlled for assessment mode, and the analyses for assessment mode controlled for promotion focus. All effects are unstandardized coefficients; ${ }^{+}$ $p<.10 ; * p<.05 ; * * p<.01$. 


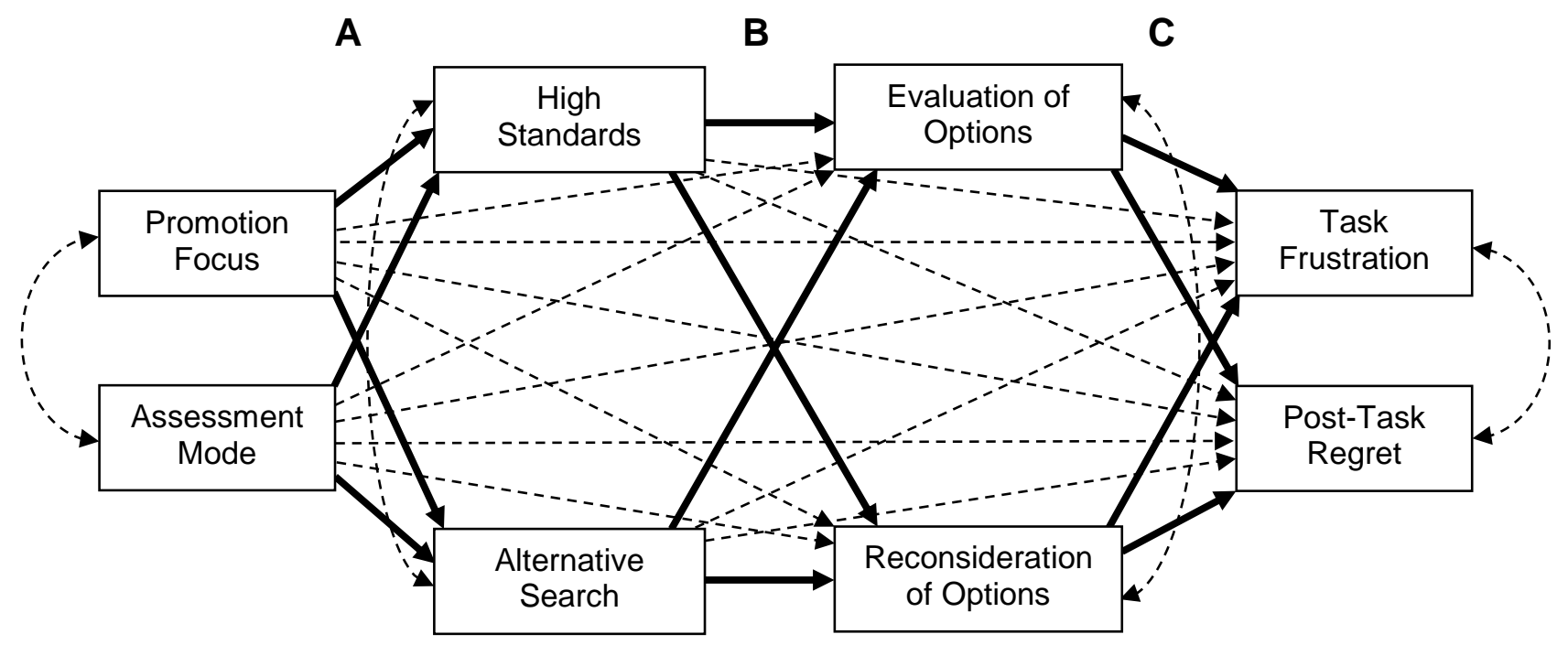

Figure 2. Path model for alternative search, evaluation of options and reconsideration of options mediating the effects of promotion and assessment on post-decision frustration and regret (Study 3). Disturbance terms have been removed for simplicity. Bolded pathways are paths for which mediation was tested; dashed lines are paths that were controlled for in all analyses. See Table 3 for estimates. 